\title{
Efficient expressions for moments of dependent random sums using copulas
}

DOI:

10.1016/j.cam.2018.12.037

\section{Document Version}

Accepted author manuscript

Link to publication record in Manchester Research Explorer

\section{Citation for published version (APA):}

Jiang, X., \& Nadarajah, S. (2019). Efficient expressions for moments of dependent random sums using copulas. Journal of Computational and Applied Mathematics, 353, 130-139. https://doi.org/10.1016/j.cam.2018.12.037

\section{Published in:}

Journal of Computational and Applied Mathematics

\section{Citing this paper}

Please note that where the full-text provided on Manchester Research Explorer is the Author Accepted Manuscript or Proof version this may differ from the final Published version. If citing, it is advised that you check and use the publisher's definitive version.

\section{General rights}

Copyright and moral rights for the publications made accessible in the Research Explorer are retained by the authors and/or other copyright owners and it is a condition of accessing publications that users recognise and abide by the legal requirements associated with these rights.

\section{Takedown policy}

If you believe that this document breaches copyright please refer to the University of Manchester's Takedown Procedures [http://man.ac.uk/04Y6Bo] or contact uml.scholarlycommunications@manchester.ac.uk providing relevant details, so we can investigate your claim.

\section{OPEN ACCESS}




\title{
Efficient expressions for moments of dependent random sums using copulas
}

\author{
by \\ Xiao Jiang and Saralees Nadarajah \\ School of Mathematics, University of Manchester, Manchester M13 9PL, UK
}

\begin{abstract}
Mao and Zhao [IMA Journal of Management Mathematics, 25, 2014, 421-433] derived the mean and variance of a dependent random sum, where the dependence was specified by a copula. Here, we derive expressions for the general moment of the dependent random sum. We also extend Mao and Zhao's results to the case that the components of the sum are not identically distributed. The practical usefulness of the results (in terms of computational time and computational accuracy) is examined by simulation.
\end{abstract}

Keywords: Farlie-Gumbel-Morgenstern copula; Insurance; Inter-arrival time; Poisson process

\section{Introduction}

Sums of a random number of random variables arise in many areas of the sciences and engineering. A prominent example is the total insurance claim over a fixed period. In this example, the number is the number of insurance claims and the variables are the amounts claimed. A related variable is the inter-arrival time between insurance claims.

There is a vast amount of literature on the distributions and moments of the random sums. Many of the papers suppose that the claims are independently and identically distributed conditioned on the number of claims. They suppose no relationship between the amounts claimed and the inter-arrival times between insurance claims. This may not be a realistic assumption. Recently, researchers have supposed that the amounts claimed and the inter-arrival times are dependent, but are marginally independently and identically distributed conditioned on the number of claims. Some papers based on this assumption are Albrecher and Teugels (2006), Asimit and Jones (2008a), Cossette et al. (2008), Marceau (2008), Ambagaspitiya (2009), Asimit and Badescu (2010) and Chueng et al. (2010). See also Asimit and Jones (2008b), Asimit et al. (2014a, 2014b), Asimit and Chen (2015) and Asimit et al. (2016).

A most recent of these papers is by Mao and Zhao (2014). They derived the first and second moments of the total insurance claim by supposing that the joint distribution of amounts claimed and the inter-arrival times is specified by a copula. They specialized their results to one of the simplest known copulas, the Farlie-Gumbel-Morgenstern (FGM) copula due to Morgenstern (1956), see also Nelsen (2006).

In this note, we extend Mao and Zhao (2014)'s results. We derive the general moment of the total insurance claim. Our results hold for a wide range of copulas not just the FGM copula. We also extend Mao and Zhao (2014)'s results to the case where the identical assumption does not hold. 
In management science and related areas, higher order moments are of interest, not just the mean and variance. Examples include: portfolio selection (Harvey et al., 2010); value-at-risk forecasting (Polanski and Stoja, 2010); market risk assessment (Sihem and Slaheddine, 2014).

The contents of this note are organized as follows. Section 2 derives the general moment of the random sums, under the assumptions given in Mao and Zhao (2014). Section 3 extends the results of Section 2 to the case that the inter-arrival times between claims are independent but not identically distributed, the amounts claimed are independent but not identically distributed, and the copulas of the joint distributions are not identical. Section 4 performs a simulation study to examine the practical values of the results in Section 2.

\section{Main results}

In this section, we suppose $N(t)$ is a renewal process with inter arrival times $W_{i}$, and $\left(X_{i}, W_{i}\right)$ are independent and identically distributed with common copula function $C$, common copula density $c$, common joint probability density function $f_{X, W}$, common joint cumulative distribution function $F_{X, W}$, common marginal probability density functions $f_{X}, f_{W}$, and common marginal cumulative distribution functions $F_{X}, F_{W}$.

Let $S_{t}=X_{1}+\cdots+X_{N(t)}$. Theorem 1 derives the $k$ th moment of $S_{t}$. It generalizes Theorems 3.1 and 4.1 in Mao and Zhao (2014). These theorems derived the first and second moments of $S_{t}$.

Theorem 1 The kth moment of $S_{t}$ can be expressed as

$$
\begin{gathered}
E\left(S_{t}^{k}\right)=\sum_{n=1}^{\infty} \sum_{k_{1}+\cdots+k_{n}=k}\left(\begin{array}{c}
k \\
k_{1} \cdots k_{n}
\end{array}\right)\left\{\int_{t-w_{i_{1}}-\cdots-w_{i_{m}}>0} F^{*(n-m)}\left(t-w_{i_{1}}-\cdots-w_{i_{m}}\right)\right. \\
\cdot \prod_{j=1}^{m}\left[g_{X^{k_{i_{j}}}}\left(w_{i_{j}}\right) f_{W}\left(w_{i_{j}}\right) d w_{i_{j}}\right] \\
-\int_{0}^{t} \int_{t-y-w_{i_{1}}-\cdots-w_{i_{m}}>0} F^{*(n-m)}\left(t-y-w_{i_{1}}-\cdots-w_{i_{m}}\right) \\
\left.\cdot \prod_{j=1}^{m}\left[g_{X^{k_{i_{j}}}}\left(w_{i_{j}}\right) f_{W}\left(w_{i_{j}}\right) d w_{i_{j}}\right] f_{W}(y) d y\right\},
\end{gathered}
$$

where

$$
g_{X^{k}}(w)=\int_{0}^{+\infty} x^{k} f_{X}(x) c\left(F_{X}(x), F_{W}(w)\right) d x
$$

and $k_{i_{1}}, \ldots, k_{i_{m}}$ denote the positive values among $k_{1}, \ldots, k_{n}$ and $w_{i_{1}}, \ldots, w_{i_{m}}$ are the corresponding ws.

Proof: Set $B_{n}=\{N(t)=n\}=\left\{W_{1}+\cdots+W_{n} \leq t\right\}-\left\{W_{1}+\cdots+W_{n+1} \leq t\right\}$ and $b_{n}=$ $\left\{w_{1}+\cdots+w_{n} \leq t\right\}-\left\{w_{1}+\cdots+w_{n+1} \leq t\right\}$. Let $I_{A}$ denote the indicator function, that is 
$I_{A}=1$ if $A$ is true and $I_{A}=0$ if $A$ is false. By the multinomial theorem,

$$
\begin{aligned}
& E\left[S_{t}^{k}\right]=E\left[\left(X_{1}+\cdots+X_{N(t)}\right)^{k}\right] \\
& =E\left\{E\left[\left(X_{1}+\cdots+X_{N(t)}\right)^{k} \mid N(t)\right]\right\} \\
& =\sum_{n=1}^{\infty} E\left[\left(X_{1}+\cdots+X_{n}\right)^{k} \mid N(t)=n\right] \operatorname{Pr}(N(t)=n) \\
& =\sum_{n=1}^{\infty} E\left[\sum_{k_{1}+\cdots+k_{n}=k}\left(\begin{array}{c}
k \\
k_{1} \cdots k_{n}
\end{array}\right) \prod_{t=1}^{n} X_{t}^{k_{t}} \mid N(t)=n\right] \operatorname{Pr}(N(t)=n) \\
& =\sum_{n=1}^{\infty} \sum_{k_{1}+\cdots+k_{n}=k}\left(\begin{array}{c}
k \\
k_{1} \cdots k_{n}
\end{array}\right) E\left[\prod_{t=1}^{n} X_{t}^{k_{t}} \mid N(t)=n\right] \operatorname{Pr}(N(t)=n) \\
& =\sum_{n=1}^{\infty} \sum_{k_{1}+\cdots+k_{n}=k}\left(\begin{array}{c}
k \\
k_{1} \cdots k_{n}
\end{array}\right) E\left[I_{B_{n}} \prod_{t=1}^{n} X_{t}^{k_{t}}\right] \\
& =\sum_{n=1}^{\infty} \sum_{k_{1}+\cdots+k_{n}=k}\left(\begin{array}{c}
k \\
k_{1} \cdots k_{n}
\end{array}\right) \int \cdots \int\left\{\prod_{b_{n}}^{m} \int x_{i_{t}}^{k_{i_{t}}} f_{X, W}\left(x_{i_{t}}, w_{i_{t}}\right) d x_{i_{t}}\right\} \\
& \cdot\left[\prod_{j=1, j \neq i_{1}, \ldots, i_{m}}^{n+1} f_{W}\left(w_{j}\right) d w_{j}\right] \\
& =\sum_{n=1}^{\infty} \sum_{k_{1}+\cdots+k_{n}=k}\left(\begin{array}{c}
k \\
k_{1} \cdots k_{n}
\end{array}\right) \int \cdots \int\left\{\prod_{b_{n}}^{m} \int x_{i_{t}}^{k_{i_{t}}} c\left(F_{X}\left(x_{i_{t}}\right), F_{W}\left(w_{i_{t}}\right)\right) f_{X}\left(x_{i_{t}}\right) d x_{i_{t}}\right\} \\
& \cdot\left[\prod_{j=1}^{n+1} f_{W}\left(w_{j}\right) d w_{j}\right] \\
& =\sum_{n=1}^{\infty} \sum_{k_{1}+\cdots+k_{n}=k}\left(\begin{array}{c}
k \\
k_{1} \cdots k_{n}
\end{array}\right) \int \cdots \int\left\{\prod_{b_{n}}^{m} g_{X^{k_{i_{t}}}}\left(w_{i_{t}}\right)\right\}\left[\prod_{j=1}^{n+1} f_{W}\left(w_{j}\right) d w_{j}\right] \\
& =\sum_{n=1}^{\infty} \sum_{k_{1}+\cdots+k_{n}=k}\left(\begin{array}{c}
k \\
k_{1} \cdots k_{n}
\end{array}\right) \int_{w_{1}+\cdots+w_{n} \leq t}\left\{\prod_{t=1}^{m} g_{X^{k_{i}}}\left(w_{i_{t}}\right)\right\}\left[\prod_{j=1}^{n+1} f_{W}\left(w_{j}\right) d w_{j}\right] \\
& -\sum_{n=1}^{\infty} \sum_{k_{1}+\cdots+k_{n}=k}\left(\begin{array}{c}
k \\
k_{1} \cdots k_{n}
\end{array}\right) \int_{w_{1}+\cdots+w_{n+1} \leq t}\left\{\prod_{t=1}^{m} g_{X^{k_{i}}}\left(w_{i_{t}}\right)\right\}\left[\prod_{j=1}^{n+1} f_{W}\left(w_{j}\right) d w_{j}\right] .
\end{aligned}
$$

The two integrals in (2) reduce to

$$
\int_{t-w_{i_{1}}-\cdots-w_{i_{m}}>0} F^{*(n-m)}\left(t-w_{i_{1}}-\cdots-w_{i_{m}}\right) \prod_{j=1}^{m}\left[g_{X^{k_{i_{j}}}}\left(w_{i_{j}}\right) f_{W}\left(w_{i_{j}}\right) d w_{i_{j}}\right]
$$

and

$\left.\int_{0}^{t} \int_{t-y-w_{i_{1}}-\cdots-w_{i_{m}}>0} F^{*(n-m)}\left(t-y-w_{i_{1}}-\cdots-w_{i_{m}}\right) \prod_{j=1}^{m}\left[g_{X^{k_{i_{j}}}}\left(w_{i_{j}}\right) f_{W}\left(w_{i_{j}}\right) d w_{i_{j}}\right] f_{W}(y) d y\right\}$. 
Hence, the result.

Corollaries 1 and 2 specialize Theorem 1 for $k=3$, 4. These corollaries can be used to compute among others the skewness and kurtosis of $S_{t}$.

Corollary 1 The third moment of $S_{t}$ can be expressed as

$$
\begin{aligned}
& E\left(S_{t}^{3}\right)=3 \sum_{n=1}^{\infty} \int_{0}^{t} F^{*(n-1)}(t-w) g_{X^{3}}(w) f_{W}(w) d w \\
& +9 \sum_{n=1}^{\infty} \int_{0}^{t} \int_{0}^{t-w_{1}} F^{*(n-2)}\left(t-w_{1}-w_{2}\right) g_{X}\left(w_{1}\right) g_{X^{2}}\left(w_{2}\right) f_{W}\left(w_{1}\right) f_{W}\left(w_{2}\right) d w_{2} d w_{1} \\
& +9 \sum_{n=1}^{\infty} \int_{0}^{t} \int_{0}^{t-w_{1}} F^{*(n-2)}\left(t-w_{1}-w_{2}\right) g_{X^{2}}\left(w_{1}\right) g_{X}\left(w_{2}\right) f_{W}\left(w_{1}\right) f_{W}\left(w_{2}\right) d w_{2} d w_{1} \\
& +6 \sum_{n=1}^{\infty} \int_{0}^{t} \int_{0}^{t-w_{1}} \int_{0}^{t-w_{1}-w_{2}} F^{*(n-3)}\left(t-w_{1}-w_{2}\right) \prod_{j=1}^{3}\left[g_{X}\left(w_{j}\right) f_{W}\left(w_{j}\right)\right] d w_{3} d w_{2} d w_{1} \\
& -3 \sum_{n=1}^{\infty} \int_{0}^{t} \int_{0}^{t-y} F^{*(n-1)}(t-y-w) g_{X^{3}}(w) f_{W}(w) f_{W}(y) d w d y \\
& -9 \sum_{n=1}^{\infty} \int_{0}^{t} \int_{0}^{t-y} \int_{0}^{t-y-w_{1}} F^{*(n-2)}\left(t-y-w_{1}-w_{2}\right) g_{X}\left(w_{1}\right) g_{X^{2}}\left(w_{2}\right) \\
& -9 \sum_{n=1}^{\infty} \int_{0}^{t} \int_{0}^{t-y} \int_{0}^{t-y-w_{1}} F^{*(n-2)}\left(t-y-w_{1}-w_{2}\right) g_{X^{2}}\left(w_{1}\right) g_{X}\left(w_{2}\right) \\
& -6 \sum_{n=1}^{\infty} \int_{0}^{t} \int_{0}^{t-y} \int_{0}^{t-y-w_{1}} \int_{0}^{t-y-w_{1}-w_{2}} F^{*(n-3)}\left(t-y-w_{1}-w_{2}-w_{3}\right) \\
& \cdot \prod_{j=1}^{3}\left[g_{X}\left(w_{j}\right) f_{W}\left(w_{j}\right)\right] f_{W}(y) d w_{3} d w_{2} d w_{1} d y . \\
& f_{W}(y) w_{2} d w_{1} d y \\
& f_{W}(y) d w_{2} d w_{1} d y
\end{aligned}
$$


Corollary 2 The fourth moment of $S_{t}$ can be expressed as

$$
\begin{aligned}
& E\left(S_{t}^{4}\right)=4 \sum_{n=1}^{\infty} \int_{0}^{t} F^{*(n-1)}(t-w) g_{X^{4}}(w) f_{W}(w) d w \\
& +36 \sum_{n=1}^{\infty} \int_{0}^{t} \int_{0}^{t-w_{1}} F^{*(n-2)}\left(t-w_{1}-w_{2}\right) g_{X^{2}}\left(w_{1}\right) g_{X^{2}}\left(w_{2}\right) f_{W}\left(w_{1}\right) f_{W}\left(w_{2}\right) d w_{2} d w_{1} \\
& +24 \sum_{n=1}^{\infty} \int_{0}^{t} \int_{0}^{t-w_{1}} F^{*(n-2)}\left(t-w_{1}-w_{2}\right) g_{X}\left(w_{1}\right) g_{X^{3}}\left(w_{2}\right) f_{W}\left(w_{1}\right) f_{W}\left(w_{2}\right) d w_{2} d w_{1} \\
& +24 \sum_{n=1}^{\infty} \int_{0}^{t} \int_{0}^{t-w_{1}} F^{*(n-2)}\left(t-w_{1}-w_{2}\right) g_{X^{3}}\left(w_{1}\right) g_{X}\left(w_{2}\right) f_{W}\left(w_{1}\right) f_{W}\left(w_{2}\right) d w_{2} d w_{1} \\
& +48 \sum_{n=1}^{\infty} \int_{0}^{t} \int_{0}^{t-w_{1}} \int_{0}^{t-w_{1}-w_{2}} F^{*(n-3)}\left(t-w_{1}-w_{2}-w_{3}\right) g_{X^{2}}\left(w_{1}\right) g_{X}\left(w_{2}\right) g_{X}\left(w_{3}\right) \\
& \cdot f_{W}\left(w_{1}\right) f_{W}\left(w_{2}\right) f_{W}\left(w_{3}\right) d w_{3} d w_{2} d w_{1} \\
& +48 \sum_{n=1}^{\infty} \int_{0}^{t} \int_{0}^{t-w_{1}} \int_{0}^{t-w_{1}-w_{2}} F^{*(n-3)}\left(t-w_{1}-w_{2}-w_{3}\right) g_{X}\left(w_{1}\right) g_{X^{2}}\left(w_{2}\right) g_{X}\left(w_{3}\right) \\
& \cdot f_{W}\left(w_{1}\right) f_{W}\left(w_{2}\right) f_{W}\left(w_{3}\right) d w_{3} d w_{2} d w_{1} \\
& +48 \sum_{n=1}^{\infty} \int_{0}^{t} \int_{0}^{t-w_{1}} \int_{0}^{t-w_{1}-w_{2}} F^{*(n-3)}\left(t-w_{1}-w_{2}-w_{3}\right) g_{X}\left(w_{1}\right) g_{X}\left(w_{2}\right) g_{X^{2}}\left(w_{3}\right) \\
& \cdot f_{W}\left(w_{1}\right) f_{W}\left(w_{2}\right) f_{W}\left(w_{3}\right) d w_{3} d w_{2} d w_{1} \\
& +24 \sum_{n=1}^{\infty} \int_{0}^{t} \int_{0}^{t-w_{1}} \int_{0}^{t-w_{1}-w_{2}} \int_{0}^{t-w_{1}-w_{2}-w_{3}} F^{*(n-4)}\left(t-w_{1}-w_{2}-w_{3}-w_{4}\right) \\
& \cdot \prod_{j=1}^{4}\left[g_{X}\left(w_{j}\right) f_{W}\left(w_{j}\right)\right] d w_{4} d w_{3} d w_{2} d w_{1} \\
& -4 \sum_{n=1}^{\infty} \int_{0}^{t} \int_{0}^{t-y} F^{*(n-1)}(t-y-w) g_{X^{4}}(w) f_{W}(w) f_{W}(y) d w d y \\
& -36 \sum_{n=1}^{\infty} \int_{0}^{t} \int_{0}^{t-y} \int_{0}^{t-y-w_{1}} F^{*(n-2)}\left(t-y-w_{1}-w_{2}\right) g_{X^{2}}\left(w_{1}\right) g_{X^{2}}\left(w_{2}\right) \\
& \cdot f_{W}\left(w_{1}\right) f_{W}\left(w_{2}\right) f_{W}(y) d w_{2} d w_{1} d y \\
& -24 \sum_{n=1}^{\infty} \int_{0}^{t} \int_{0}^{t-y} \int_{0}^{t-y-w_{1}} F^{*(n-2)}\left(t-y-w_{1}-w_{2}\right) g_{X}\left(w_{1}\right) g_{X^{3}}\left(w_{2}\right) \\
& \cdot f_{W}\left(w_{1}\right) f_{W}\left(w_{2}\right) f_{W}(y) d w_{2} d w_{1} d y \\
& -24 \sum_{n=1}^{\infty} \int_{0}^{t} \int_{0}^{t-y} \int_{0}^{t-y-w_{1}} F^{*(n-2)}\left(t-y-w_{1}-w_{2}\right) g_{X^{3}}\left(w_{1}\right) g_{X}\left(w_{2}\right) \\
& \cdot f_{W}\left(w_{1}\right) f_{W}\left(w_{2}\right) f_{W}(y) d w_{2} d w_{1} d y
\end{aligned}
$$




$$
\begin{gathered}
-48 \sum_{n=1}^{\infty} \int_{0}^{t} \int_{0}^{t-y} \int_{0}^{t-y-w_{1}} \int_{0}^{t-y-w_{1}-w_{2}} F^{*(n-3)}\left(t-y-w_{1}-w_{2}-w_{3}\right) \\
\cdot g_{X^{2}}\left(w_{1}\right) g_{X}\left(w_{2}\right) g_{X}\left(w_{3}\right) \\
\cdot f_{W}\left(w_{1}\right) f_{W}\left(w_{2}\right) f_{W}\left(w_{3}\right) f_{W}(y) d w_{3} d w_{2} d w_{1} d y \\
-48 \sum_{n=1}^{\infty} \int_{0}^{t} \int_{0}^{t-y} \int_{0}^{t-y-w_{1}} \int_{0}^{t-y-w_{1}-w_{2}} F^{*(n-3)}\left(t-y-w_{1}-w_{2}-w_{3}\right) \\
\cdot g_{X}\left(w_{1}\right) g_{X^{2}}\left(w_{2}\right) g_{X}\left(w_{3}\right) \\
\cdot f_{W}\left(w_{1}\right) f_{W}\left(w_{2}\right) f_{W}\left(w_{3}\right) f_{W}(y) d w_{3} d w_{2} d w_{1} d y \\
\sum_{n=1}^{\infty} \int_{0}^{t} \int_{0}^{t-y} \int_{0}^{t-y-w_{1}} \int_{0}^{t-y-w_{1}-w_{2}} F^{*(n-3)}\left(t-y-w_{1}-w_{2}-w_{3}\right) \\
\cdot g_{X}\left(w_{1}\right) g_{X}\left(w_{2}\right) g_{X^{2}}\left(w_{3}\right) \\
\cdot f_{W}\left(w_{1}\right) f_{W}\left(w_{2}\right) f_{W}\left(w_{3}\right) f_{W}(y) d w_{3} d w_{2} d w_{1} d y \\
-24 \sum_{n=1}^{\infty} \int_{0}^{t} \int_{0}^{t-y} \int_{0}^{t-y-w_{1}} \int_{0}^{t-y-w_{1}-w_{2}} \int_{0}^{t-y-w_{1}-w_{2}-w_{3}} F^{*(n-4)}\left(t-y-w_{1}-w_{2}-w_{3}-w_{4}\right) \\
\cdot \prod_{j=1}^{4}\left[g_{X}\left(w_{j}\right) f_{W}\left(w_{j}\right)\right] f_{W}(y) d w_{4} d w_{3} d w_{2} d w_{1} d y .
\end{gathered}
$$

Corollary 3 specializes Theorem 1 to the case that $N(t)$ is a Poisson process with rate parameter $\lambda$.

Corollary 3 Suppose $\{N(t)\}$ is a Poisson process with rate parameter $\lambda$. Then the $k$ th moment of $S_{t}$ can be expressed as

$$
\begin{gathered}
E\left(S_{t}^{k}\right)=\sum_{n=1}^{\infty} \sum_{k_{1}+\cdots+k_{n}=k}\left(\begin{array}{c}
k \\
k_{1} \cdots k_{n}
\end{array}\right) \frac{\lambda^{m}}{(n-m) !} \\
\left\{\int_{t-w_{i_{1}}-\cdots-w_{i_{m}}>0} \gamma\left(n-m, \lambda\left(t-w_{i_{1}}-\cdots-w_{i_{m}}\right)\right)\right. \\
\cdot \exp \left(-\lambda \sum_{j=1}^{m} w_{i_{j}}\right) \prod_{j=1}^{m}\left[g_{X^{k_{j}}}\left(w_{i_{j}}\right) d w_{i_{j}}\right] \\
-\lambda \int_{0}^{t} \int_{t-y-w_{i_{1}}-\cdots-w_{i_{m}}>0} \gamma\left(n-m, \lambda\left(t-y-w_{i_{1}}-\cdots-w_{i_{m}}\right)\right) \\
\left.\cdot \exp \left(-\lambda y-\lambda \sum_{j=1}^{m} w_{i_{j}}\right) \prod_{j=1}^{m}\left[g_{X^{k_{i_{j}}}}\left(w_{i_{j}}\right) d w_{i_{j}}\right] d y\right\}
\end{gathered}
$$

where $g_{X^{k}}(\cdot)$ is given by (1), $\gamma(a, x)$ denotes the incomplete gamma function defined by

$$
\gamma(a, x)=\int_{0}^{x} t^{a-1} \exp (-t) d t
$$

and $k_{i_{1}}, \ldots, k_{i_{m}}$ denote the positive values among $k_{1}, \ldots, k_{n}$ and $w_{i_{1}}, \ldots, w_{i_{m}}$ are the corresponding ws. 
Nadarajah (2015) showed that a wide range of bivariate copulas (including the FGM copula) can be expressed as

$$
c(u, v)=\sum_{i=1}^{n} \alpha_{i} a_{i} b_{i} u^{a_{i}-1} v^{b_{i}-1}
$$

for $n \geq 1$ an integer and $\left\{\left(\alpha_{i}, a_{i}, b_{i}\right): i \geq 1\right\}$ some real numbers. Using (3), we can write

$$
g_{X^{k}}(w)=\sum_{i=1}^{n} \alpha_{i} a_{i} b_{i} F_{W}^{b_{i}-1}(w) \int_{0}^{+\infty} x^{k} f_{X}(x) F_{X}^{a_{i}-1}(x) d x .
$$

If $a_{i}$ are positive integers and if $X_{1}, \ldots, X_{a_{i}}$ are independent and identical copies of $X$ then (4) can be further rewritten as

$$
g_{X^{k}}(w)=\sum_{i=1}^{n} \alpha_{i} b_{i} F_{W}^{b_{i}-1}(w) E\left[Z_{i}^{k}\right]
$$

where $Z_{i}=\max \left(X_{1}, \ldots, X_{a_{i}}\right)$.

\section{An extension}

Theorem 2 extends Theorem 1 to the case that $\left(X_{1}, W_{1}\right),\left(X_{2}, W_{2}\right), \ldots$ are independent but not identical. We suppose that $\left(X_{i}, W_{i}\right)$ has copula function $C^{(i)}$, copula density $c^{(i)}$, joint probability density function $f_{X_{i}, W_{i}}$, joint cumulative distribution function $F_{X_{i}, W_{i}}$, marginal probability density functions $f_{X_{i}}, f_{W_{i}}$, and marginal cumulative distribution functions $F_{X_{i}}$, $F_{W_{i}}$.

Theorem 2 The kth moment of $S_{t}$ can be expressed as

$$
\begin{gathered}
E\left(S_{t}^{k}\right)=\sum_{n=1}^{\infty} \sum_{k_{1}+\cdots+k_{n}=k}\left(\begin{array}{c}
k \\
k_{1} \cdots k_{n}
\end{array}\right)\left\{\int_{t-w_{i_{1}}-\cdots-w_{i_{m}}>0} F^{*(n-m)}\left(t-w_{i_{1}}-\cdots-w_{i_{m}}\right)\right. \\
\cdot \prod_{j=1}^{m}\left[g_{X^{k_{i_{j}}}}^{\left(i_{j}\right)}\left(w_{i_{j}}\right) f_{W_{i_{j}}}\left(w_{i_{j}}\right) d w_{i_{j}}\right] \\
-\int_{0}^{t} \int_{t-y-w_{i_{1}}-\cdots-w_{i_{m}}>0} F^{*(n-m)}\left(t-y-w_{i_{1}}-\cdots-w_{i_{m}}\right) \\
\left.\cdot \prod_{j=1}^{m}\left[g_{X^{k_{i_{j}}}}^{\left(i_{i}\right)}\left(w_{i_{j}}\right) f_{W_{i_{j}}}\left(w_{i_{j}}\right) d w_{i_{j}}\right] f_{W_{n+1}}(y) d y\right\},
\end{gathered}
$$

where

$$
g_{X^{k}}^{(i)}(w)=\int_{0}^{+\infty} x^{k} f_{X_{i}}(x) c^{(i)}\left(F_{X_{i}}(x), F_{W_{i}}(w)\right) d x
$$

and $k_{i_{1}}, \ldots, k_{i_{m}}$ denote the positive values among $k_{1}, \ldots, k_{n}$ and $w_{i_{1}}, \ldots, w_{i_{m}}$ are the corresponding ws. 
Proof: Define $B_{n}, b_{n}$ and $I_{A}$ as in the proof of Theorem 1. By the multinomial theorem,

$$
\begin{aligned}
& E\left[S_{t}^{k}\right]=E\left[\left(X_{1}+\cdots+X_{N(t)}\right)^{k}\right] \\
& =\sum_{n=1}^{\infty} E\left[\left(X_{1}+\cdots+X_{n}\right)^{k} \mid N(t)=n\right] \operatorname{Pr}(N(t)=n) \\
& =\sum_{n=1}^{\infty} \sum_{k_{1}+\cdots+k_{n}=k}\left(\begin{array}{c}
k \\
k_{1} \cdots k_{n}
\end{array}\right) E\left[\prod_{t=1}^{n} X_{t}^{k_{t}} \mid N(t)=n\right] \operatorname{Pr}(N(t)=n) \\
& =\sum_{n=1}^{\infty} \sum_{k_{1}+\cdots+k_{n}=k}\left(\begin{array}{c}
k \\
k_{1} \cdots k_{n}
\end{array}\right) E\left[I_{B_{n}} \prod_{t=1}^{n} X_{t}^{k_{t}}\right] \\
& =\sum_{n=1}^{\infty} \sum_{k_{1}+\cdots+k_{n}=k}\left(\begin{array}{c}
k \\
k_{1} \cdots k_{n}
\end{array}\right) \int \cdots \int\left\{\prod_{b_{n}}^{m} \int x_{i_{t}}^{k_{i_{t}}} f_{X_{i_{t}}, W_{i_{t}}}\left(x_{i_{t}}, w_{i_{t}}\right) d x_{i_{t}}\right\} \\
& {\left[\prod_{j=1, j \neq i_{1}, \ldots, i_{m}}^{n+1} f_{W_{j}}\left(w_{j}\right) d w_{j}\right]} \\
& =\sum_{n=1}^{\infty} \sum_{k_{1}+\cdots+k_{n}=k}\left(\begin{array}{c}
k \\
k_{1} \cdots k_{n}
\end{array}\right) \int \cdots \int\left\{\prod_{b_{n}}^{m} \int x_{i_{t}}^{k_{i_{t}}} c^{\left(i_{t}\right)}\left(F_{X_{i_{t}}}\left(x_{i_{t}}\right), F_{W_{i_{t}}}\left(w_{i_{t}}\right)\right) f_{X_{i_{t}}}\left(x_{i_{t}}\right) d x_{i_{t}}\right\} \\
& \cdot\left[\prod_{j=1}^{n+1} f_{W_{j}}\left(w_{j}\right) d w_{j}\right] \\
& =\sum_{n=1}^{\infty} \sum_{k_{1}+\cdots+k_{n}=k}\left(\begin{array}{c}
k \\
k_{1} \cdots k_{n}
\end{array}\right) \int \cdots \int\left\{\prod_{b_{n}}^{m} g_{X^{k_{i}}}^{\left(i_{t}\right)}\left(w_{i_{t}}\right)\right\}\left[\prod_{j=1}^{n+1} f_{W_{j}}\left(w_{j}\right) d w_{j}\right] \\
& =\sum_{n=1}^{\infty} \sum_{k_{1}+\cdots+k_{n}=k}\left(\begin{array}{c}
k \\
k_{1} \cdots k_{n}
\end{array}\right) \int_{w_{1}+\cdots+w_{n} \leq t}\left\{\prod_{t=1}^{m} g_{X^{k_{i}}}^{\left(i_{t}\right)}\left(w_{i_{t}}\right)\right\}\left[\prod_{j=1}^{n+1} f_{W_{j}}\left(w_{j}\right) d w_{j}\right] \\
& -\sum_{n=1}^{\infty} \sum_{k_{1}+\cdots+k_{n}=k}\left(\begin{array}{c}
k \\
k_{1} \cdots k_{n}
\end{array}\right) \int_{w_{1}+\cdots+w_{n+1} \leq t}\left\{\prod_{t=1}^{m} g_{X^{k_{i}}}^{\left(i_{t}\right)}\left(w_{i_{t}}\right)\right\}\left[\prod_{j=1}^{n+1} f_{W_{j}}\left(w_{j}\right) d w_{j}\right] \text {. }
\end{aligned}
$$

The two integrals in (5) reduce to

$$
\int_{t-w_{i_{1}}-\cdots-w_{i_{m}}>0} F^{*(n-m)}\left(t-w_{i_{1}}-\cdots-w_{i_{m}}\right) \prod_{j=1}^{m}\left[g_{X^{k_{i_{j}}}}^{\left(i_{j}\right)}\left(w_{i_{j}}\right) f_{W_{i_{j}}}\left(w_{i_{j}}\right) d w_{i_{j}}\right]
$$

and

$$
\left.\int_{0}^{t} \int_{t-y-w_{i_{1}}-\cdots-w_{i_{m}}>0} F^{*(n-m)}\left(t-y-w_{i_{1}}-\cdots-w_{i_{m}}\right) \prod_{j=1}^{m}\left[g_{X^{k_{i_{j}}}}^{\left(i_{j}\right.}\left(w_{i_{j}}\right) f_{W_{i_{j}}}\left(w_{i_{j}}\right) d w_{i_{j}}\right] f_{W_{n+1}}(y) d y\right\} .
$$

Hence, the result.

\section{Simulation}


In this section, we examine computational efficiency of the expressions derived in Section 2. Computational efficiency is assessed in terms of time and accuracy.

Suppose $N(t)$ is a Poisson process with rate parameter $\lambda, X_{i}$ are independent and identical exponential random variables with rate parameter $\lambda$ and $\left(X_{i}, W_{i}\right)$ have the common copula function $C(u, v)=u v+\theta u v(1-u)(1-v)$, the FGM copula function. We computed $E\left(S_{t}^{k}\right)$ by simulation and using Theorem 1 for every $k=1,2, \ldots, 100$. The simulation was performed as follows:

1. simulate $N$ from a Poisson distribution with parameter $\lambda$;

2. simulate $\left(U_{1}, V_{1}\right), \ldots,\left(U_{N}, V_{N}\right)$ from the FGM copula;

3. set $X_{i}=-\frac{1}{\lambda} \log \left(1-U_{i}\right)$ and $W_{i}=-\frac{1}{\lambda} \log \left(1-V_{i}\right)$ for $i=1, \ldots, N$;

4. compute $S_{t}^{k}=\left(X_{1}+\cdots+X_{N}\right)^{k}$;

5. repeat steps 1 to 4 one million times;

6. compute the average of the values of $S_{t}^{k}$.

$E\left(S_{t}^{k}\right)$ was also computed using Theorem 1 for every $k=1,2, \ldots, 100$. The Maple software was used, please see the code listed. Maple like other algebraic manipulation packages allows for arbitrary precision. For a given value of Digits, Maple approximates $E\left(S_{t}^{k}\right)$ by truncated versions of the infinite sums in Theorem 1 and makes sure that the difference between successive truncated sums does not exceed the error specified by Digits. That is, the values computed using Maple can be considered exact up to a given decimal place.

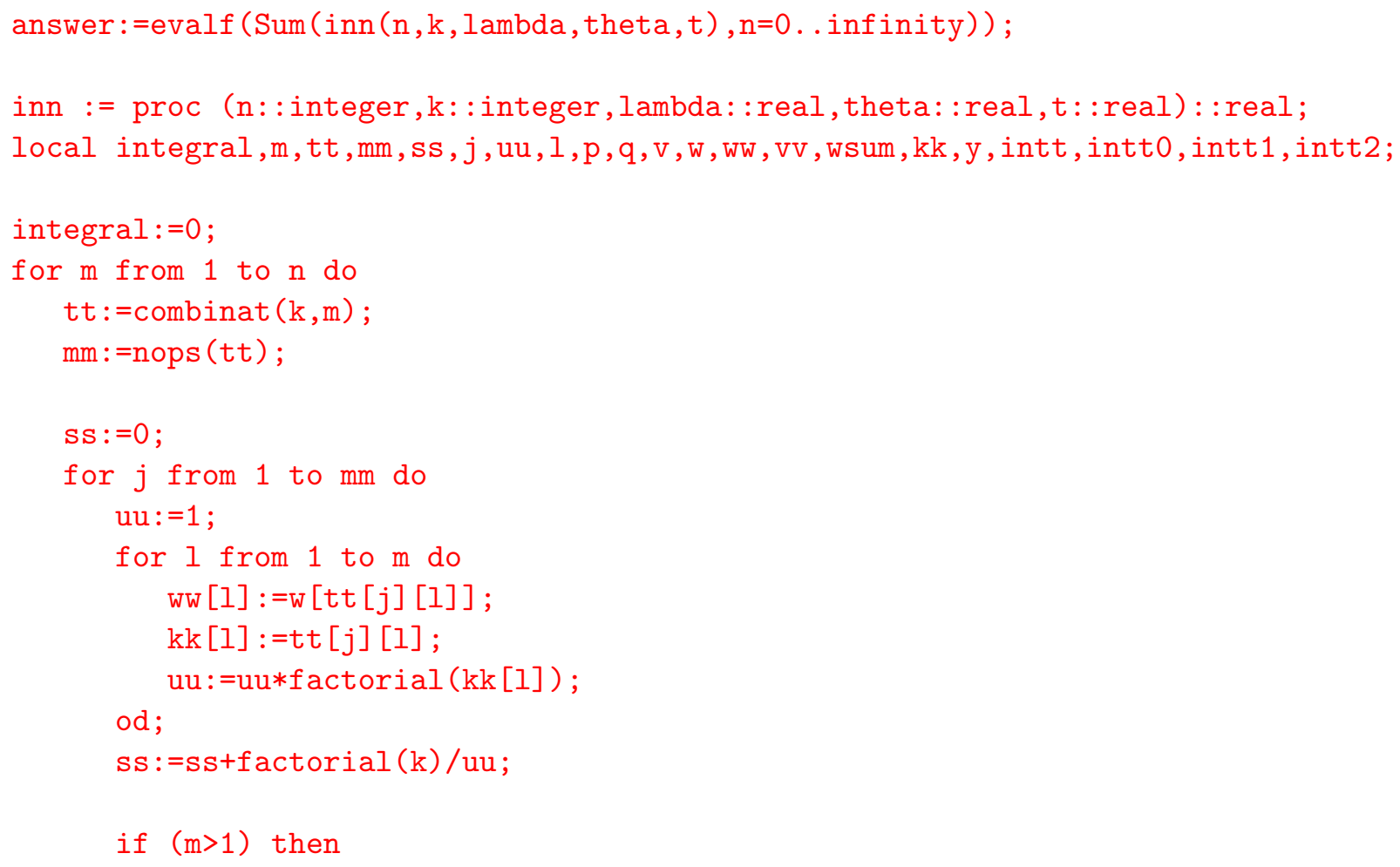




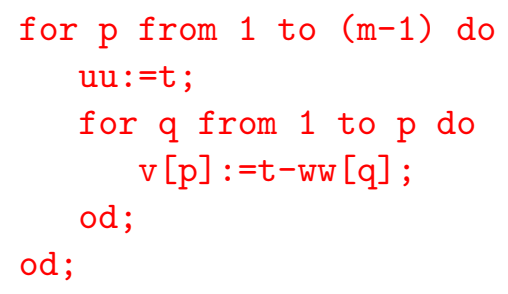


end proc;

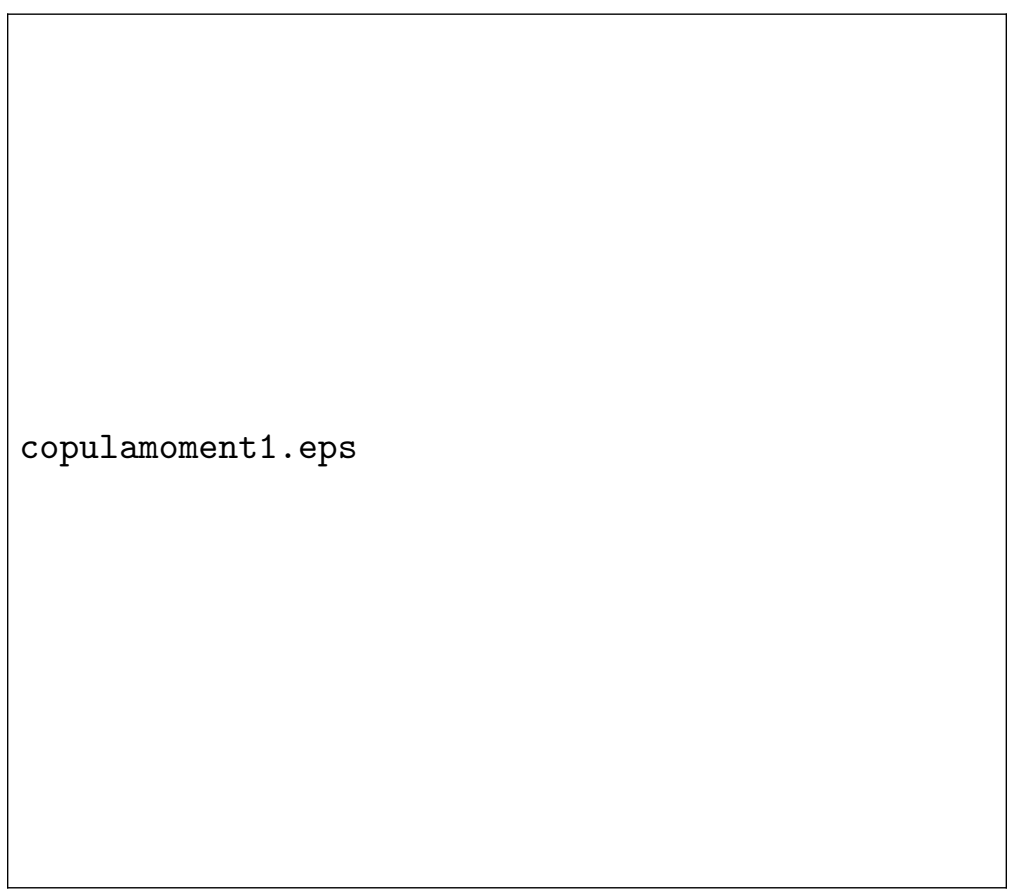

Figure 1: Central processing unit times in seconds taken to compute $E\left(S_{t}^{k}\right)$ by simulation (solid line) and by using Theorem 1 (broken line). 


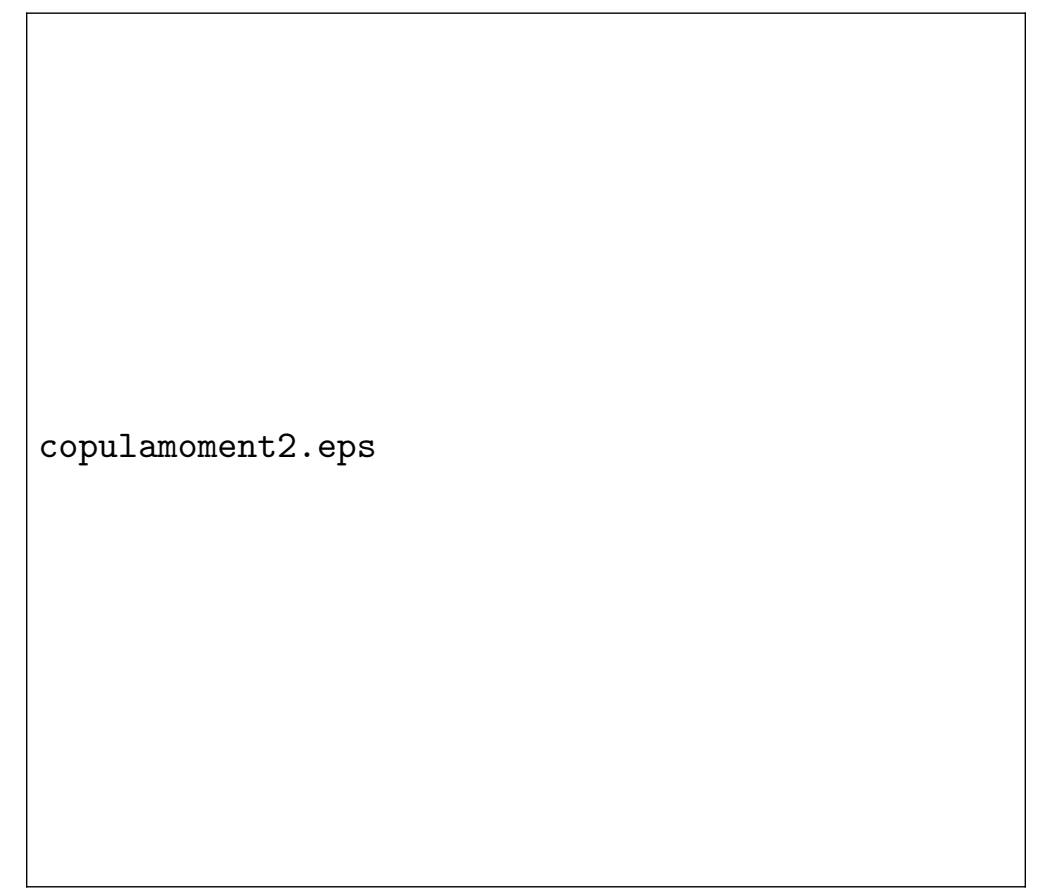

Figure 2: The exact minus the simulated values of $E\left(S_{t}^{k}\right)$ versus $k$.

The central processing unit times taken in seconds to compute $E\left(S_{t}^{k}\right)$ are plotted in Figure 1. The differences between the simulated and exact values of $E\left(S_{t}^{k}\right)$ are plotted in Figure 2. We have taken $\lambda=1, \theta=-0.6,-0.2,0.2,0.6$ and $k=1, \ldots, 100$.

We see that our expression in Theorem 1 appears little more efficient for all values of $k$ in terms of the central processing unit times and accuracy. But simulations can be sped up considerably by using a general purpose language such as Python or $\mathrm{C}++$ to give better central processing unit times and accuracy. Hence, it is difficult to say which of the two methods (using Theorem 1 or the simulation approach) is better.

In Figures 1 and 2, we have taken the copula to be the FGM copula. But the conclusion on the choice between using Theorem 1 and the simulation approach was similar for a wide range of copulas of the form (3) and for a wide range of parameter values.

\section{Acknowledgments}

The authors would like to thank the Editor and the two referees for careful reading and comments which greatly improved the paper.

\section{References}

[1] Albrecher, H. and Teugels, J. (2006). Exponential behavior in the presence of dependence in risk theory. Journal of Applied Probability, 43, 265-285. 
[2] Ambagaspitiya, R. S. (2009). Ultimate ruin probability in the Sparre Andersen model with dependent claim sizes and claim occurrence times. Insurance: Mathematics and Economics, 44, 464-472.

[3] Asimit, V. and Badescu, A. (2010). Extremes on the discounted aggregate claims in a time dependent risk model. Scandinavian Actuarial Journal, 93-104.

[4] Asimit, V. and Chen, Y. (2015). Asymptotic results for conditional measures of association of a random sum. Insurance: Mathematics and Economics, 60, 11-18.

[5] Asimit, V., Hashorva, E. and Kortschak, D. (2014a). Tail asymptotics of randomly weighted large risks. arXiv: 1405.0593

[6] Asimit, V., Hashorva, E. and Kortschak, D. (2014b). Asymptotic tail probability of randomly weighted large risks. arXiv: 1405.0593

[7] Asimit, V., Hashorva, E. and Kortschak, D. (2016). Aggregation of randomly weighted large risks. IMA Journal of Management Mathematics, doi: 10.1093/imaman/dpv020

[8] Asimit, V. and Jones, B. (2008a). Dependence and the asymptotic behavior of large claims reinsurance. Insurance: Mathematics and Economics, 43, 407-411.

[9] Asimit, V. and Jones, B. (2008b). Asymptotic tail probabilities for large claims reinsurance of a portfolio of dependent risks. ASTIN Bulletin, 38, 147-159.

[10] Chueng, E. C. K., Landriault, D., Willmot, G. E. and Woo, J. K. (2010). Structural properties of Gerber-Shiu functions in dependent Sparre Andersen models. Insurance: Mathematics and Economics, 46, 117-126.

[11] Cossette, H., Marceau, E. and Fouad, M. F. (2008). On the compound Poisson risk model with dependence based on a generalized Farlie-Gumbel-Morgenstern copula. Insurance: Mathematics and Economics, 43, 444-455.

[12] Harvey, C. R., Liechty, J. C., Liechty, M. W. and Müller, P. (2010). Portfolio selection with higher moments. Quantitative Finance, 10, 469-485.

[13] Mao, Z. -C. and Zhao, X. (2014). The expectation and variance of dependent random sums using copulas. IMA Journal of Management Mathematics, 25, 421-433.

[14] Marceau, E. (2008). On the discrete-time compound renewal risk model with dependence. Insurance: Mathematics and Economics, 44, 245-259.

[15] Morgenstern, D. (1956). Einfache beispiele zweidimensionaler verteilungen. Mitteilingsblatt für Mathematishe Statistik, 8, 234-235.

[16] Nadarajah, S. (2015). Expansions for bivariate copulas. Statistics and Probability Letters, 100, 77-84.

[17] Nelsen, R. B. (2006). An Introduction to Copulas, second edition. Springer Verlag, New York.

[18] Polanski, A. and Stoja, E. (2010). Incorporating higher moments into value-at-risk forecasting. Journal of Forecasting, 29, 523-535. 
[19] Sihem, M. and Slaheddine, H. (2014). The impact of higher order moments on market risk assessment. Procedia Economics and Finance, 13, 143-153. 